\title{
Family Physicians' Sharing of Personal Information on Facebook
}

\author{
Kamila Premji $^{1^{\star}}$, Raywat Deonandan ${ }^{2}$ and Andrea Nwosu ${ }^{2}$ \\ ${ }^{1}$ Department of Family Medicine, University of Ottawa, 43 Bruyère Street, Ottawa, Canada. \\ ${ }^{2}$ Faculty of Health Sciences, University of Ottawa, Canada.
}

\begin{abstract}
Authors' contributions
This work was carried out in collaboration between all authors. Author KP performed the concept, methodology, manuscript writing, review of final manuscript. Author RD performed the data analysis, manuscript writing, review of final manuscript. Author AN performed the data collection, review of final manuscript.
\end{abstract}

Research Article

Received $28^{\text {th }}$ May 2013

Accepted 25 $5^{\text {th }}$ July 2013

Published $14^{\text {th }}$ September 2013

\section{ABSTRACT}

Aims: Online social networks, such as Facebook, are growing in popularity amongst physicians, and represent a potential avenue for the compromising of their privacy and professional boundaries. We sought to determine the extent to which family doctors are sharing personal information on Facebook.

Study Design: Observational.

Place and Duration of Study: Ottawa, Ontario, Canada. Data was collected between May and August of 2012.

Methodology: From the website of the College of Physicians and Surgeons of Ontario, all 1000 family physicians active in the Ottawa region were identified. A database was then created of subjects' publicly shared information (i.e., information available to the general public) for those with accessible profiles on Facebook. Chi square and t-tests were performed to explore demographic patterns for those with viewable profiles; binary logistic regression was used to identify factors associated with Facebook visibility.

Results: While only $10.2 \%$ of family physicians had publicly viewable profiles, $81.4 \%$ of those featured a visible profile photo; $91.2 \%$ could be messaged directly by any member of the public; a majority shared limited personal information, including recent online activities and place of education; $24 \%$ shared their place of work; $15 \%$ had visible lists of family members; and $14 \%$ disclosed their relationship status. From logistic regression, there were no significant factors associated with whether a physician was viewable on 
Facebook.

Conclusion: While a minority of physicians have a publicly accessible Facebook profile, those that do are sharing personal information that may expose them to unwanted intrusions into their personal lives and unexpected patient interactions outside of the office. Physicians should be aware of options for making their online information less publicly accessible.

Keywords: Online social networking; social media; internet; professionalism; boundaries.

\section{INTRODUCTION}

As online social networking (OSN) via platforms such as Facebook, Twitter, and Linkedin continues to grow, issues pertaining to the ethics and boundaries of doctor-patient communication over OSN have likewise become increasingly prominent in the medical literature. Several studies have highlighted concerns about the type of information posted by physicians and medical trainees for public viewing [1-6]. Family physicians, who often develop long-term relationships with patients and their families, have been identified as common targets for patient-initiated communication over OSN [7].

The potential for physician-patient interaction outside of the office, and the potential for community access to physicians' personal information, are issues rife with ethical implications. A survey of American physicians and medical students [7] found that most respondents felt interacting with patients via OSN is not only ethically unacceptable, but offered little value to the patient-physician communication dynamic. Prising open physicians' accessibility in forums outside of the office may present unwelcomed opportunities for unprofessionalism, miscommunication, and the general discomfort of physicians as their personal domains are gradually impinged upon by patients' concerns.

Various medical professional bodies have begun to respond to the literature on professionalism and OSN. In the U.K., the General Medical Council's regulations for doctors' use of social medial came into effect on April 22, 2013 [8]. The British Medical Association has also published guidelines on the use of social media by doctors and medical students [9]. In the United States, the American Medical Association and Federation of State Medical Boards have published professionalism guidelines on the subject [10-11]. In Canada, the Canadian Medical Association (CMA), College of Physicians and Surgeons of British Columbia, and the College of Physicians and Surgeons of New Brunswick, have published similar guidelines [12-14]. In the Canadian literature, the CMAJ recently published a series of commentaries on social media [15-17].

Despite growing recognition by various national professional bodies of the need to guide physicians on appropriate use of social media, there is little research paying specific attention to family physicians who, as noted earlier, have been identified as being particularly vulnerable to patient contact over OSN platforms.

We sought to further explore the accessibility of family physicians to the general public over OSN and in turn help guide family physician-specific professionalism policy and education. To do so, we used Facebook to examine the extent to which a regional sample of Canadian family physicians are publicly accessible over OSN, and the extent to which boundaries may be compromised based on public visibility of personal information. 


\section{METHODOLOGY}

An application was submitted to the Research Ethics Board of the University of Ottawa, but review was waived due to our protocol meeting the requirements for REB exemption.

From the publicly available website of the College of Physicians and Surgeons of Ontario, the following data were extracted for all 1000 physicians (exact number) listed as practicing family medicine in Ottawa as of May, 2012: name, workplace postal code, sex, and medical school graduation year.

A dummy Facebook account was created with no connections to other Facebook profiles ("friends") or networks. Using that account, a dedicated search was performed for each physician identified to locate a matching profile on Facebook. In cases where the identity of the profile owner could not be precisely matched to the physician's name, additional Google searches were performed for the subject's location, image and alternate spellings of the name to determine with some certainty whether a found Facebook profile was indeed that of the physician in question.

Once a Facebook profile match was confirmed, the following information was collected for each subject: visibility of photos; likelihood that the profile photo was that of the physician in question (confirmed via Google search); and the public visibility of family members, hobbies, relationship status, religious and political views, email address, telephone number, instant messaging address, personal website, date of birth, ethnicity, sexual orientation, list of Facebook friends, subject's workplace, posts on the Facebook "wall", comments on those posts, list of the subject's recent Facebook activities, and alma mater. We also determined whether the profile was accepting friend requests (determined from the existence of a "friend" button); and whether a message could be sent to the profile (determined from the existence of a "message" button). Because we used the dummy account for all of our searches, only data that Facebook users had made available to the general public could be collected.

Using SPSS version 20, univariate descriptive analyses were performed on both the full set of 1000 physicians, and the subset of subjects with viewable Facebook profiles, to explore the demographic nature of the population. Bivariable analyses (chi-square and t-tests) were performed to determine the roles of gender and years since graduation in the expression of online Facebook behaviours. Lastly, a binary logistic regression analysis was performed to identify factors associated with the visibility of a Facebook profile. For the latter, the outcome variable was whether or not the profile was viewable by the general public; the modeled putative explanatory factors were all the demographic variables collected from the profiles.

\section{RESULTS}

From the exactly 1000 subjects identified from the CPSO website, 102 were matched with publicly viewable Facebook profiles. Table 1 summarizes the cascading sizes of our subsamples.

Our initial sample of 1000 subjects featured an average work experience (years since graduation) of 23.1 years, ranging from 2 to 62 years. While $52.5 \%$ were female, the women had less work experience $(p<0.001)$ with an average of 19.6 years since graduation, compared to 27.0 years for men. 
Table 1. Cascading sample sizes of our initial sample and subsequent sub-samples

\begin{tabular}{ll}
\hline Sample & N \\
\hline All subjects from CPSO Ottawa database & 1000 \\
All subjects for whom a Facebook profile was found matching their name & 219 \\
Subjects with matching Facebook profiles that we are reasonably certain are theirs & 107 \\
Facebook profiles which were publicly viewable & 102 \\
Publicly viewable profiles that can be messaged by anyone & 93 \\
Publicly viewable profiles with profile photos & 92 \\
Profiles whose profile pictures we are reasonably certain depict the named subject & 84 \\
\hline
\end{tabular}

In terms of listed postal codes, a plurality of subjects was clustered around eight hospital areas; in declining order: the Montfort Hospital, the Elizabeth Bruyere Centre, Ottawa Civic Hospital, Ottawa Health Sciences Centre, Westboro Travel Medicine Clinic, Irving Greenberg Family Cancer Centre, University of Ottawa Health Services, and the National Defense Headquarters of Canada.

Amongst those with publicly viewable Facebook profiles, the mean work experience was 17.4 years, ranging from $3-50$ years, comprised of $55.9 \%$ females. As with the initial sample, the female subjects in this subset had significantly less work experience than the males (13.5 years compared to 22.3 years; $p<0.001$ ).

No one had made available the following information: political and religious views, sexual orientation, date of birth, direct contact information (email, telephone, or instant messaging), website, or mailing address.

However, as summarized in Table 2, a fair number had made other personal information publicly available, most commonly the ability to be sent a message, the ability to be added as a friend, recent Facebook activities, place of education, and an identifying profile photo.

Table 2. Public information on physicians' Facebook profiles

\begin{tabular}{lll}
\hline $\begin{array}{l}\text { Information publicly available on subject's Facebook } \\
\text { profile }\end{array}$ & $\begin{array}{l}\text { Number of } \\
\text { subjects }\end{array}$ & Percentage \\
\hline Publicly viewable photo of the subject & 83 & 81.4 \\
Relationship status given & 14 & 13.7 \\
Visible friends list & 78 & 76.5 \\
Place of work named & 24 & 23.5 \\
Visible hobbies list & 29 & 28.4 \\
Can be added as a friend & 98 & 96.1 \\
Can be sent a message & 93 & 91.2 \\
Visible list of family members & 15 & 14.7 \\
Recent Facebook activities are visible & 84 & 82.4 \\
Recent wall posts are visible & 31 & 30.4 \\
Comments on wall posts are visible & 23 & 22.5 \\
Place of education is identified & 84 & 82.4 \\
\hline
\end{tabular}

Among the 14 subjects who indicated their relationship status, 11 claimed to be married, 2 to be single, and 1 indicated being "in a relationship". Those 14 subjects had a significantly greater mean years of work experience than those who did not indicate their status (25.6 years vs 16.1 years; $p=0.006$ ). 
The results of binary logistic regression suggest that none of the factors measured is statistically associated with whether a physician's Facebook profile is publicly viewable. Nor, among those that were viewable, is there a strong factor predictive of whether a physician's profile can be messaged.

\section{DISCUSSION}

Family physicians have been identified as being particularly vulnerable to patient-initiated communication outside the workplace [7]. Given that Canada is among the top 20 most "wired" nations in the world, [18] and Ottawa among its most wired communities [19], the public accessibility of personal information for family physicians in this community via OSN is of relevance to all caregivers nationally and worldwide. This study examines family physician professionalism and boundaries in the context of online social media.

In terms of detecting physicians' vulnerability to privacy intrusion, our findings were both encouraging and troubling. On the one hand, fewer than $10 \%$ of physicians in the Ottawa sample had a viewable Facebook profile; and none of those shared contact information or profoundly personal details, such as political and religious views. In light of a study showing that $51 \%$ of Canadian physicians are Facebook users [20], this suggests that a majority of Ottawa family physicians are making use of their Facebook privacy settings. However, on the other hand, almost all of the viewable profiles included information on hobbies, recent Facebook activities, and places of education. Such information contributes to a "profiling" of the physician, which may constitute a type of privacy intrusion. Furthermore, almost all were contactable, either through a message or a "Friend" request, by any member of the general public. Since the time of this study, this has become an even more potentially problematic issue, as Facebook has, as of December 2012, modified its privacy policy to allow for anyone on Facebook, regardless of his/her privacy settings, be accessible via messaging by anyone else with a profile on the website.

The commonness of visibility of family and friends lists is a further pathway to the subjects' lifestyles. A smaller though relevant proportion of viewable profiles also featured the subject's relationship status, information that empowers both fraudsters and perhaps even overly curious patients or community members seeking a connection with the subject outside the confines of their professional relationship. It is interesting to note that no associations were found between gender and a tendency to share any information online, though the female subjects with viewable profiles were more likely to have fewer years of experience. While myths proliferate concerning the gender make-up of the typical OSN user, in Canada there is an almost even split between the sexes, with Facebook users being $53 \%$ female [21]. Moreover, using years since graduation as a proxy measure for age, we found no significant relationship between age and the degree of information sharing on Facebook, with the exception that doctors with more experience were more likely to share their relationship status. This is important because OSN usage is considered to be preferred by younger users (half of Canadian users being in the 25-44 years age range [22], and because previous research on medical professionalism and OSN has suggested a generational effect, such that younger Facebook users post more actively on Facebook [5,23].

There are several limitations to our study that may be addressed by future research in this area. By limiting our OSN platform to Facebook, we may be underestimating the availability of publicly visible personal physician data online. As demonstrated in the recent Canadian Medical Association survey of Canadian physicians, only $51 \%$ reported having a Facebook account [20], and in a recent U.S. study that included a search of all web-based platforms, 
researchers were able to find personal information online for $32.4 \%$ of physicians [24]. It is possible that Canadian (or Ottawa-area) physicians are more aware of privacy, professionalism, and boundary considerations when engaging in OSN, but it is also possible that by limiting our study to Facebook, we did not cast a wide enough net. By limiting our data collection to practicing physicians, we also did not capture physicians-in-training. This younger, "Facebook generation" of doctors has been the focus of many startling studies out of the U.S. demonstrating a high prevalence of inappropriate personal data posted online by medical trainees.[1,3,5-6] The CMA survey demonstrated that only $18 \%$ of Canadian medical students agree that social media is of little value in day-to-day medical practice, compared with $47.5 \%$ of practicing physicians [20]. In light of these data, it would be interesting to see how Canadian trainees protect their privacy and professionalism online in comparison to their American counterparts. Finally, by limiting our study to a specific region and specialty, our data is limited in its generalizability.

The methodology employed in this study brings to light important ethical considerations when conducting research that involves accessing public databases where the participants' identities are known, reflecting a wider societal debate about the appropriateness of mining large, ostensibly public datasets containing personal information meant for use within limited social networks. As noted earlier, to ensure ethical rigor in our methods, we sought approval from a local Research Ethics Board, conformed to their prescribed standards for identity protection, and have presented here only non-identifying data. The philosophy of research ethics in general may need to evolve in light of the availability of these types of data. Convery and Cox (2012) elaborate on this discussion and propose ethical guidelines for internet-based research while recognizing that no single set of guidelines can be applied to all scenarios [25], and Janssens and Kraft (2012) delve into the ethical issues around methodological limitations for clinical research gathered through OSN [26]. Researchers will need to become increasingly cognizant of the ethical nuances of exploring social media data.

\section{CONCLUSION}

Our results suggest that a reassuring majority of family physicians in Ottawa are not accessible to the general public via Facebook. However, our findings also indicate that a significant minority (one in ten) are vulnerable to breaches in professionalism and boundaries on this particular OSN platform. Many users are likely unaware that their information is publicly viewable, and that there are steps they can take to make their OSN usage more private and secure. While the privacy setting options on Facebook and similar sites are seemingly in a constant state of evolution, they are nevertheless sufficiently sensitive for a user to select from a variety of levels of public visibility. With the accelerating popularity of OSN, there is an ongoing need to incorporate its suggested usage into formal professional education programs and policymaking efforts.

\section{CONSENT}

Not applicable as only publicly available data was used for this study. 


\section{ETHICAL APPROVAL}

An application was submitted to the Research Ethics Board of the University of Ottawa, but review was waived due to our protocol meeting the requirements for REB exemption.

\section{COMPETING INTERESTS}

Authors have declared that no competing interests exist.

\section{REFERENCES}

1. Thompson LA, Dawson K, Ferdig R, Black EW, Boyer J, Coutts J, et al. The intersection of online social networking with medical professionalism. J Gen Int Med. 2008;23(7):954-7.

2. Gorrindo T, Groves JE. Intersection of online social networking with medical professionalism: Can medicine police the Facebook boom? J Gen Int Med. 2008;23(12):2155.

3. Chretien KC, Greysen SR, Chretien J, KindT. Online posting of unprofessional content by medical students. JAMA. 2009;302(12):1309-15.

4. Ahmed $\mathrm{O}$, Wardle $\mathrm{A}$, Caesar R. Online professionalism and Facebook - falling through the generation gap. Med Teach. 2012;e1-8.

5. Macdonald J, Sohn S, Ellis P. Privacy, professionalism and Facebook: a dilemma for young doctors. Med Ed. 2010;44:805-13.

6. Black E, Thompson L, Duff WP, Dawson K, Saliba H, Paradise Black NM. Revisiting social network utilization by physicians-in-training. J Grad Med Ed. 2010;289-93.

7. Bosslet G, Torke AM, Hickman SE, Colin LT, Helft PR. The patient-doctor relationship and online social networks: Results of a national Survey. J Gen Intern Med. $2011 ; 26(10): 1168-74$.

8. General Medical Council (GMC). 2013. Doctors' use of social media. Accessed 26 May 2013.

Available: www.gmc-uk.org/Doctors use of social media.pdf 51448306.pdf.

9. British Medical Association (BMA). Using social media: practical and ethical guidance for doctors and medical students. Accessed 26 May 2013. Available: bma.org.uk//media/Files/PDFs/.../Ethics/socialmediaguidance.pdf

10. American Medical Association (AMA). 2011. AMA policy: Professionalism in the use of social media. Accessed 2 Dec 2012. Available: http://www.amaassn.org/ama/pub/meeting/professionalism-social-media.shtml.

11. Federation of State Medical Boards (FSMB). Model policy guidelines for the appropriate use of social media and social networking in medical practice. Accessed 2 Dec 2012. Available: http://www.fsmb.org/pdf/pub-social-media-guidelines.pdf.

12. Canadian Medical Association (CMA). 2011. Social media and Canadian physicians issues and rules of engagement. Accessed 23 Dec 2012.

Available: http://www.cma.ca/advocacy/social-media-canadian-physicians.

13. College of Physicians and Surgeons of British Columbia (CPSBC). 2010. Professional Standards and Guidelines: Social Media and Online Networking Forums. Accessed 21 Dec 2012.

Available:https://www.cpsbc.ca/files/u6/Social-Media-and-Online-Networking-

Forums.pdf. 
14. College of Physicians and Surgeons of New Brunswick. 2010. Selected commentaries: Facebook. Accessed 23 Dec 2012.

Available: http://www.cpsnb.org/english/Guidelines/Facebook.htm.

15. Collier R. Professionalism: logging on to tell your doctor off. CMAJ.2012; 184(12):E629-30.

16. Collier R. Professionalism: social media mishaps. CMAJ. 2012;184(12):E627-8.

17. Collier R. Professionalism: social media outreach. CMAJ. 2012;184(11):E587-8.

18. Internet World Stats. Top 50 countries with the highest internet penetration rate. Accessed 21 Dec 2012. Available: http://www.internetworldstats.com/top25.htm.

19. Statistics Canada. Canadian Internet Use Survey. May 10, 2010. Accessed 21 Dec, 2012. Available: http://www.statcan.gc.ca/daily-quotidien/100510/dg100510a-eng.htm.

20. Canadian Medical Association. Canadian physicians and social media: Activity and attitudes. 2011. Accessed 23 Dec 2012. Available: http://www.slideshare.net/prich/canadian-physicians-and-social-media-a-survey.

21. Socialbakers.com. Canada Facebook Statistics. Accessed 21 Dec 2012.

Available: http://www.socialbakers.com/facebook-statistics/canada.

22. Royal Pingdom. Report: social network demographics in 2012. Accessed 21 Dec 2012.

Available:http://royal.pingdom.com/2012/08/21/report-social-network-demographics-in$2012 /$

23. Read B. Think Before You Share. Students' online socializing can have unintended consequences. Chron High Educ. 2006;52(20):38-41.

24. Mostaghimi A, Crotty B, Landon BE. The availability and nature of physician information on the internet. J Gen Intern Med. 2010;25(11):152-6.

25. Convery I, Cox D. A review of research ethics in internet-based research. Practitioner Research in Higher Education. Accessed 19 Jul 2012;6(1):50-7.

Available: http://194.81.189.19/ojs/index.php/prhe/article/viewFile/100/192

26. Janssens ACJW, Kraft P. Research Conducted Using Data Obtained through Online Communities: Ethical Implications of Methodological Limitations. PLOS Medicine. 2012;9(10). e1001328. doi:10.1371/journal.pmed.1001328.

(c) 2014 Premji et al.; This is an Open Access article distributed under the terms of the Creative Commons Attribution License (http://creativecommons.org/licenses/by/3.0), which permits unrestricted use, distribution, and reproduction in any medium, provided the original work is properly cited.

Peer-review history:

The peer review history for this paper can be accessed here: http://www.sciencedomain.org/review-history. php?iid=215\&id=12\&aid=2017 